(c) American Dairy Science Association, 2005.

\title{
Relationship Among Trans and Conjugated Fatty Acids and Bovine Milk Fat Yield Due to Dietary Concentrate and Linseed Oil
}

\author{
J. J. Loor, ${ }^{\star}$ A. Ferlay, A. Ollier, M. Doreau, and Y. Chilliard \\ Herbivore Research Unit INRA-Theix, 63122 St.-Genes Champanelle, France
}

\begin{abstract}
Effects on fatty acid profiles and milk fat yield due to dietary concentrate and supplemental 18:3n-3 were evaluated in 4 lactating Holstein cows fed a low- (35:65 concentrate:forage; L) or high- $(65: 35 ; \mathrm{H})$ concentrate diet without (LC, HC) added oil or with linseed oil (LCO, $\mathrm{HCO})$ at $3 \%$ of DM. A $4 \times 4$ Latin square with four 4 wk periods was used. Milk yield and dry matter intake averaged 26.7 and $20.2 \mathrm{~kg} / \mathrm{d}$, respectively, across treatments. Plasma acetate and $\beta$-hydroxybutyrate decreased, whereas glucose, nonesterified fatty acids, and leptin increased with high-concentrate diets. Milk fat percentage was lower in cows fed high-concentrate diets (2.31 vs. 3.38), resulting in decreases in yield of 11 (HC) and $42 \%$ (HCO). Reduced yields of 8:0-16:0 and cis9$18: 1$ fatty acids accounted for 69 and $17 \%$, respectively, of the decrease in milk fat yield with HC vs. LC (-90 $\mathrm{g} / \mathrm{d}$ ), and for 26 and $33 \%$, respectively, of the decrease with $\mathrm{HCO}$ vs. LCO $(-400 \mathrm{~g} / \mathrm{d})$. Total trans-18:1 yield increased by 25 (HCO) and 59 (LCO) g/d with oil addition. Trans 10-18:1 yield was 5-fold greater with highconcentrate diets. Trans11-18:1 increased by 13 (HCO) and 19 (LCO) g/d with oil addition. Trans13+14-18:1 yield increased by 9 (HCO) and 18 (LCO) g/d with linseed oil. Yield of total conjugated linoleic acids (CLA) in milk averaged $6 \mathrm{~g} / \mathrm{d}$ with LC or HC compared with $14 \mathrm{~g} / \mathrm{d}$ with LCO or HCO. Cis9,trans11-CLA yield was not affected by concentrate level but increased by $147 \%$ in response to oil. Feeding oil increased yields of trans11,cis13-, trans11,trans13-, and trans,trans-CLA, primarily with LCO. Trans10,cis12-CLA yield (average of $0.08 \mathrm{~g} / \mathrm{d}$ ) was not affected by treatments. Yield of trans11,cis 15-18:2 was $1 \mathrm{~g} / \mathrm{d}$ in cows fed LC or HC and $10 \mathrm{~g} / \mathrm{d}$ with LCO or HCO. Yields of cis9,trans11-18:2, cis 9,trans 12-18:2, and cis9,trans 13-18:2 were positively correlated ( $\mathrm{r}=0.74$ to 0.94$)$ with yields of trans $11-18: 1$, trans12-18:1, and trans13+14-18:1, respectively.
\end{abstract}

\footnotetext{
Received May 4, 2004.

Accepted October 22, 2004.

Corresponding author: Y. Chilliard; e-mail: Yves.Chilliard@ clermont.inra.fr.

*Present address: Department of Animal Sciences, University of Illinois, 206 ERML, Urbana, IL, 61801 (jloor@uiuc.edu).
}

Plasma concentrations of biohydrogenation intermediates with concentrate or linseed oil level followed similar changes as those in milk fat. Milk fat depression was observed when HC induced an increase in trans 10 18:1 yield. A correlation of 0.84 across 31 comparisons from 13 published studies, including the present one, was found among the increase in percentage of trans 10 18:1 in milk fat and decreased milk fat yield. We observed, however, more drastic milk fat depression when HCO increased yields of total trans-18:1, trans 11 ,cis 15 $18: 2$, trans isomers of $18: 3$, and reduced yields of $18: 0$ plus cis9-18:1.

(Key words: high concentrate, linseed oil, milk conjugated linoleic acids, trans fatty acids)

Abbreviation key: $\mathbf{L C}=$ low-concentrate diet without linseed oil, $\mathbf{H C}=$ high-concentrate diet without linseed oil, $\mathbf{L C O}=$ low-concentrate diet with linseed oil, $\mathbf{H C O}=$ high-concentrate diet with linseed oil, CLA = conjugated linoleic acids.

\section{INTRODUCTION}

Diets with a high concentrate:forage ratio fed with unsaturated oils reduce milk fat production to the largest extent (review by Bauman and Griinari, 2003). Biohydrogenation of polyunsaturated fatty acids in the rumen is reduced with high-concentrate diets causing accumulation of trans-18:1 isomers (Kalscheur et al., 1997; Doreau et al., 1999). In cows fed high-concentrate diets without (Piperova et al., 2002) or with (Griinari et al., 1998; Piperova et al., 2000; Peterson et al., 2003) vegetable oils rich in linoleic acid, trans 10-18:1 percentage in milk fat was as high or higher than trans1118:1. Trans7,cis9-18:2 and trans10,cis12-18:2, along with cis9,trans11-18:2, also increased primarily when the high-concentrate diet was supplemented with oil high in linoleic acid (Piperova et al., 2000).

Experiments assessing the effects of concentrate:forage ratio on milk fat composition have used corn silage alone, alfalfa hay alone, corn plus alfalfa or grass silage, or corn plus mixtures of small cereal grain silages (e.g., barley and triticale) as the forage source, with corn grain being the primary source of starch in the concentrate mixture (Kalscheur et al., 1997; Griinari et al., 
1998; Piperova et al., 2000; Peterson et al., 2003). Highlinoleic acid oils (e.g., corn or soybean oil) were the preferred substrate for biohydrogenation. Type of forage and supplemental unsaturated fatty acids alter trans-18:1 and conjugated linoleic acid (CLA) isomers in milk fat to varying extents in cows (Chilliard et al., 2001) or goats (Chilliard et al., 2003a). Differences in starch source may account for some of the observed variation in the milk fat depression response in cows fed high-concentrate diets (Bauman and Griinari, 2003).

To our knowledge, there are no published studies comparing level of concentrate:forage ratio and linseed oil (a source of $\alpha$-linolenic acid) on milk composition. The present study examined the effects of feeding highconcentrate diets containing wheat as the primary starch source fed alone or in combination with linseed oil and grass hay as the sole forage on milk fat yield, blood plasma metabolites, and profiles of trans-18:1 and CLA isomers in blood plasma and milk fat. Results pertaining to ruminal digestion (Ueda et al., 2003) and duodenal flows and digestibility of fatty acids (Loor et al., 2004) have already been reported.

\section{MATERIALS AND METHODS}

\section{Animals and Diets}

Four peak-lactation multiparous Holstein cows with cannulas in the rumen and duodenum were used in a $4 \times 4$ Latin square design with factorial arrangement of treatments during four 4-wk periods to evaluate responses to feeding diets with a low (35:65) or high (65:35) concentrate to forage ratio without (LC, HC) added oil or with linseed oil (LCO, HCO) supplemented at 3\% of DM (Ueda et al., 2003; Loor et al., 2004). Average BW and DIM for cows at the onset of the experiment was $658 \pm 13 \mathrm{~kg}$ and $71 \pm 16 \mathrm{~d}$. The sole forage was long-cut grass hay and the concentrate mixture was based primarily on ground wheat, rapeseed, sunflower meal, and wheat bran. Details of ingredient, chemical composition, and fatty acid profiles of the diet have been presented previously (Loor et al., 2004). By design, diets were not isoenergetic but were close to being isonitrogenous. Cows were housed in a tie-stall barn during the experiment. The concentrate mixture with or without linseed oil was prepared daily and, along with the forage, was offered in equal amounts at 0900, 1330, and $1700 \mathrm{~h}$ for ad libitum consumption. The first $5 \mathrm{~d}$ of each experimental period were used as a transition between treatments. Cows were milked at 0600 and $1700 \mathrm{~h}$.

\section{Sampling, Measurements, and Analyses}

Milk production and DMI were recorded daily throughout the experiment. Milk was sampled at each milking during the last $5 \mathrm{~d}$ of wk 4 . One $50-\mathrm{mL}$ aliquot from each of these milkings containing potassium bichromate (Merck, Fontenay-Sus-Bois, France) was stored at $4^{\circ} \mathrm{C}$ until analyzed for fat, protein, and lactose by infrared analysis with a 3-channel spectrophotometer (AOAC, 1997; CILAL, Theix, France). Additional 3$\mathrm{mL}$ aliquots from 2 consecutive milkings on the last day of wk 4 were collected and stored at $-20^{\circ} \mathrm{C}$ until the end of the experiment before lyophilization (Thermovac TM-20, Froilabo, Ozoir-la-Ferriere, France) and fatty acid analysis. These samples were composited based on a.m. and p.m. milk production. Data on milk production and DMI were averaged over the last $5 \mathrm{~d}$ of wk 4 before statistical analysis.

For plasma total fatty acid analysis, blood samples $(50 \mathrm{~mL}$ ) were obtained with heparinized (150 USP) Vacutainer tubes (CML, Nemours, France) from the jugular vein at $0830 \mathrm{~h}$ on the last day of wk 4 . For plasma metabolite analysis, an additional $10 \mathrm{~mL}$ of blood was collected from the jugular and abdominal mammary vein by venipuncture using a Vacutainer tube containing EDTA $(0.47 \mathrm{~mol} / \mathrm{L})$. Blood was centrifuged at $3000 \times \mathrm{g}$ for $15 \mathrm{~min}$ for harvesting plasma. Plasma was stored at $-20^{\circ} \mathrm{C}$ until analyzed for fatty acids and metabolites.

Plasma concentrations of metabolites were determined as described by Ferlay and Chilliard (1999) with an ELAN autoanalyzer (Merck-Clevenot S.A., Nogentsur-Marne, France), by spectrophotometric assays using specific kits (Glucose RTU, BioMerieux, Marcy-l'Etoile, France; Urea, kit no. 489 620, Boehringer-Mannheim, Meylan, France; nonesterified fatty acids, NEFA C, WAKO, Unipath S.A., Dardilly, France; free and esterified cholesterol kits, Biotrol Diagnostic, Chennevieres-les-Louvres, France; phospholipids PAP 150, BioMerieux; triglycerides PAP 150, BioMerieux; acetate, kit no. 0148 261, Boehringer-Mannheim; lactate PAP, BioMerieux). $\beta$-Hydroxybutyrate was analyzed with an automated micromethod (Ferlay and Chilliard, 1999). Insulin was determined by radioimmunoassay using a commercial kit (INSI-PR RIA, CIS Bio International, Gif-sur-Yvette, France). Leptin was determined using a specific radioimmunoassay, as described by Delavaud et al. (2002). Intraassay CV for BHBA, lactate, glucose, free glycerol, NEFA, total cholesterol, free cholesterol, phospholipid, triglyceride, insulin, and leptin were 4.1, $0.5,0.5,1.7,0.5,1.7,2.4,2,2.4,6.6$, and $6 \%$, respectively. Interassay CV for BHBA, lactate, glucose, free glycerol, NEFA, total cholesterol, free cholesterol, phospholipid, triglyceride, insulin, and leptin were 4, 8, 2, $4,7,2.3,2.5,3.2,5,11.2$, and $9 \%$, respectively.

Plasma total lipids were extracted with chloroform/ methanol (2:1, vol/vol; Loor et al., 2002a,b). Fatty acids in lyophilized milk were directly methylated with $1 \mathrm{~mL}$ 
of $2 \mathrm{~N}$ methanolic $\mathrm{NaOCH}_{3}$ at room temperature for $20 \mathrm{~min}$, followed by $1 \mathrm{~mL}$ of $14 \%$ boron trifluoride in methanol at room temperature for $20 \mathrm{~min}$ (Christie et al., 2001). Fatty acids in plasma lipids were methylated with $2 \mathrm{~mL}$ of $0.5 \mathrm{~N} \mathrm{NaOCH}_{3}$ at $50^{\circ} \mathrm{C}$ for $30 \mathrm{~min}$, followed by $2 \mathrm{~mL}$ of $14 \%$ boron trifluoride in methanol at $50^{\circ} \mathrm{C}$ for $30 \mathrm{~min}$ (Loor et al., 2002a,b). In all cases, fatty acid methyl esters were recovered in $1 \mathrm{~mL}$ of hexane. Tricosanoate (Sigma, Saint-Quentin Fallavier, France) was used as the internal standard. Samples were injected by autosampler into a Trace-GC 2000 Series gas chromatograph equipped with a flame ionization detector (Thermo Finnigan, Les Ulis, France). Methyl esters from all samples were separated on a $100 \mathrm{~m} \times 0.25 \mathrm{~mm}$ i.d. fused silica capillary column (CP-Sil 88, Chrompack, Middelburg, The Netherlands). Identification of 18:1, 18:2, CLA, and 18:3 isomers and odd and branched-chain fatty acids was as described in Loor et al. (2004). A butter reference standard (CRM 164; Commission of the European Communities, Community Bureau of Reference, Brussels, Belgium) was used to estimate correction factors for short-chain (4:0 to 10:0) fatty acids.

For fatty acid analysis ( 0.5 to $1 \mu \mathrm{L}$ of methyl esters in hexane injected at a 50:1 split ratio), the injector temperature was maintained at $250^{\circ} \mathrm{C}$ and the detector temperature was maintained at $255^{\circ} \mathrm{C}$. The initial oven temperature was held at $70^{\circ} \mathrm{C}$ for $1 \mathrm{~min}$, increased $5^{\circ} \mathrm{C} / \mathrm{min}$ to $100^{\circ} \mathrm{C}$ (held for $2 \mathrm{~min}$ ), then increased at $10^{\circ} \mathrm{C} / \mathrm{min}$ to $175^{\circ} \mathrm{C}$ (held for $40 \mathrm{~min}$ ), and $5^{\circ} \mathrm{C} / \mathrm{min}$ to a final temperature of $225^{\circ} \mathrm{C}$ (held for $15 \mathrm{~min}$ ). Hydrogen was the carrier gas. Injector pressure was held constant at $158.6 \mathrm{kPa}$. Satisfactory separations of cis- and trans18:1, nonconjugated 18:2, and CLA isomers were obtained with a single chromatographic run (see Figure 1 in Loor et al., 2004).

\section{Statistical Analysis}

Data for forage and concentrate intake, milk production and composition, fatty acids in plasma, and fatty acids in milk fat are reported as least squares means \pm SEM. Data were analyzed as a Latin square with factorial arrangement of treatments using the MIXED procedure of SAS (SAS Inst., Inc., Cary, NC). The statistical model included: cow, period, forage level, oil supplementation level, forage $\times$ oil interaction, and residual error. Fixed effects included period, forage level, oil supplementation level, and forage $\times$ oil interaction. Cow was the random effect. Compound symmetry was the covariate structure used. Nonlinear regression analysis and ANOVA were used to examine relationships among selected fatty acids, or between selected fatty acids and milk fat percentage.
Overall differences between treatment means were considered to be significant when $P \leq 0.05$. Interactions for level of concentrate and oil were considered significant at $P \leq 0.10$ to guard against Type II error. This was deemed appropriate because of the low power of the experimental design to test for interactions rather than main effects, combined with the a priori expectation for interactions.

\section{RESULTS}

\section{DMI and Milk Production and Composition}

Average DMI and milk, protein, and lactose yields during the last $5 \mathrm{~d}$ of wk 4 did not differ in response to concentrate or oil level (Table 1). Protein content decreased with LCO compared to LC but increased with $\mathrm{HCO}$ compared to $\mathrm{HC}$ (concentrate $\times$ oil interaction, $P$ $<0.05)$. Feeding high-concentrate diets substantially reduced milk fat percentage and yield $(-28 \%)$. The reduction in milk fat yield was less pronounced in cows fed HC compared to LC $(-11 \%)$ than HCO compared to LCO $(-42 \%)$ (concentrate $\times$ oil interaction, $P=0.07)$.

\section{Blood Plasma Metabolite Concentrations}

In cows fed high-concentrate diets, acetate and BHBA concentrations in jugular plasma were lower $(P<0.05)$ compared with low-concentrate diets (Table 2). In contrast, feeding high-concentrate diets increased $(P<$ 0.05 ) concentrations of glucose, glycerol, and NEFA. Concentration of leptin tended $(P=0.10)$ to be greater in cows fed the high-concentrate diets. Linseed oil supplementation resulted in concentrations of glucose, total cholesterol, free cholesterol, phospholipid, and urea that were $4,27,24,24$, and $23 \%$, respectively, greater $(P<0.05)$.

Jugular-mammary venous differences for acetate and BHBA decreased $(P<0.05)$ in response to feeding highconcentrate diets, but the jugular-mammary venous difference for glucose was increased (Table 2). Jugularmammary venous difference for NEFA was near zero when $\mathrm{HC}$ or LC were fed but was negative $(P<0.05)$ when $\mathrm{HCO}$ or LCO were fed. Jugular-mammary venous difference for insulin tended $(P=0.07)$ to be greater in response to feeding high-concentrate diets.

\section{Plasma Fatty Acid Concentrations}

Concentration of total fatty acids in jugular blood plasma averaged $2883 \mu \mathrm{g} / \mathrm{mL}$ in response to linseed oil $(P<0.05)$ compared with $2114 \mu \mathrm{g} / \mathrm{mL}$ for unsupplemented diets (Table 3). When cows were fed high-concentrate diets, concentration of iso-15, anteiso- 15 , iso16 , and cis 10-17:1 was lower $(P<0.05)$. Cows fed linseed 

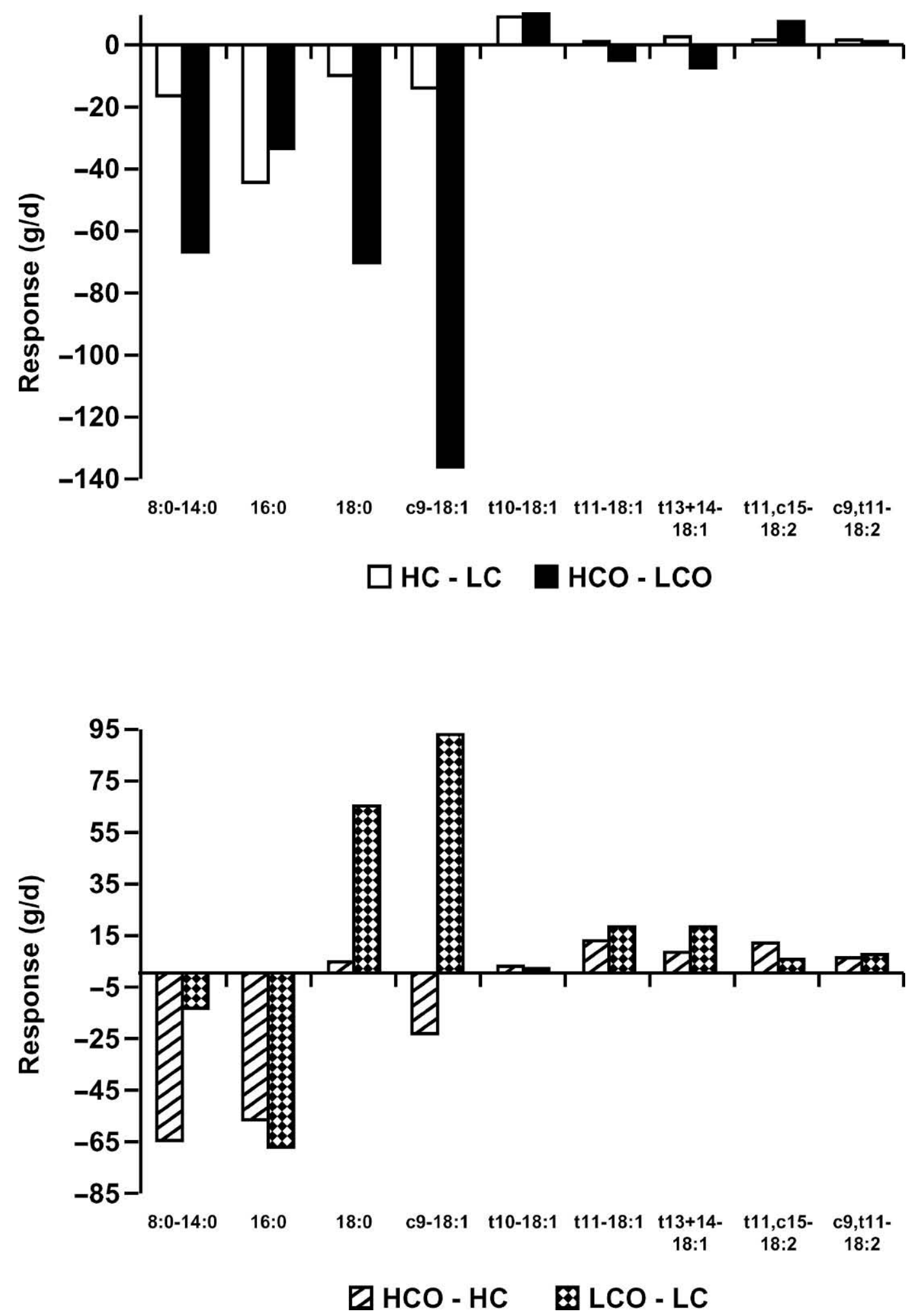

Figure 1. Secretion response of selected fatty acid isomers in response to feeding the high-concentrate vs. low-concentrate (HC-LC), high-concentrate plus linseed oil vs. low-concentrate plus linseed oil (HCO-LCO), high-concentrate plus linseed oil vs. high-concentrate (HCO-HC), or the low-concentrate plus linseed oil diet vs. low-concentrate diet (LCO-LC). 
Table 1. Dry matter intake and milk production and composition in Holstein cows fed a high- $(\mathrm{H})$ or low(L) concentrate diet without supplemental oil (HC, LC), or supplemented at 3\% of DM with linseed oil (HCO, $\mathrm{LCO})$.

\begin{tabular}{|c|c|c|c|c|c|c|c|c|}
\hline \multirow[b]{2}{*}{ Variable } & \multicolumn{4}{|c|}{ Diets } & \multirow[b]{2}{*}{ SEM } & \multicolumn{3}{|c|}{ Effect $^{1}$} \\
\hline & $\mathrm{LC}$ & LCO & $\mathrm{HC}$ & $\mathrm{HCO}$ & & Conc & Oil & Conc $\times$ Oil \\
\hline \multicolumn{9}{|l|}{ DMI, kg/d } \\
\hline Forage & 13.0 & 12.4 & 6.9 & 6.4 & 0.5 & 0.01 & 0.23 & 0.98 \\
\hline Concentrate & 7.4 & 7.2 & 13.5 & 14.0 & 0.6 & 0.01 & 0.75 & 0.48 \\
\hline Total & 20.4 & 19.6 & 20.4 & 20.4 & 1.0 & 0.65 & 0.64 & 0.65 \\
\hline Milk, kg/d & 24.2 & 27.7 & 28.8 & 26.2 & 2.6 & 0.50 & 0.85 & 0.20 \\
\hline \multicolumn{9}{|c|}{ Composition, \% } \\
\hline Fat & 3.30 & 3.45 & 2.40 & 2.22 & 0.25 & 0.01 & 0.95 & 0.52 \\
\hline Protein & 3.10 & 2.95 & 3.03 & 3.21 & 0.13 & 0.12 & 0.81 & 0.01 \\
\hline Lactose & 4.50 & 4.83 & 4.76 & 4.78 & 0.13 & 0.42 & 0.19 & 0.24 \\
\hline \multicolumn{9}{|l|}{ Yield, kg/d } \\
\hline Fat & 0.79 & 0.95 & 0.70 & 0.55 & 0.08 & 0.01 & 0.91 & 0.07 \\
\hline Protein & 0.75 & 0.81 & 0.88 & 0.84 & 0.09 & 0.29 & 0.85 & 0.49 \\
\hline Lactose & 1.09 & 1.34 & 1.38 & 1.25 & 0.13 & 0.41 & 0.64 & 0.14 \\
\hline
\end{tabular}

${ }^{1} P$ value for the effect of concentrate level (Conc), oil, and the interaction of concentrate and oil.

oil had greater concentrations of 15:0, anteiso-15:0, cis916:1, cis 10-17:1, 19:0, and 20:5n-3 $(P<0.05)$. A significant concentrate $\times$ oil interaction $(P<0.05)$ was found for the concentration of 14:0 and 18:0 in plasma.

\section{Plasma 18:1 Isomer Concentrations}

Total trans-18:1 in plasma increased $(P=0.01)$ substantially with linseed oil, and more markedly when it

Table 2. Plasma metabolite ( $\mathrm{mmol} / \mathrm{L}$ ) and hormone concentrations, and apparent mammary uptake in Holstein cows fed a high- (H) or low(L) concentrate diet without supplemental oil (HC, LC), or supplemented at 3\% of DM with linseed oil (HCO, LCO).

\begin{tabular}{|c|c|c|c|c|c|c|c|c|}
\hline \multirow[b]{2}{*}{ Variable } & \multicolumn{4}{|c|}{ Diets } & \multirow[b]{2}{*}{ SEM } & \multicolumn{3}{|c|}{ Effect $^{1}$} \\
\hline & $\mathrm{LC}$ & $\mathrm{LCO}$ & $\mathrm{HC}$ & $\mathrm{HCO}$ & & Conc & Oil & Conc $\times$ Oil \\
\hline \multicolumn{9}{|l|}{ Jugular concentration } \\
\hline Acetate & 0.983 & 0.783 & 0.433 & 0.416 & 0.150 & 0.01 & 0.45 & 0.49 \\
\hline$\beta$-hydroxybutyrate & 0.373 & 0.340 & 0.235 & 0.217 & 0.040 & 0.01 & 0.49 & 0.74 \\
\hline Lactate & 0.964 & 0.815 & 0.991 & 0.626 & 0.203 & 0.63 & 0.15 & 0.53 \\
\hline Glucose & 3.25 & 3.40 & 3.55 & 3.70 & 0.07 & 0.01 & 0.05 & 0.91 \\
\hline Total cholesterol & 4.39 & 5.69 & 3.93 & 4.86 & 0.41 & 0.14 & 0.02 & 0.66 \\
\hline Free cholesterol & 0.943 & 1.21 & 0.852 & 1.02 & 0.08 & 0.12 & 0.02 & 0.59 \\
\hline Phospholipid & 2.11 & 2.73 & 2.05 & 2.42 & 0.18 & 0.32 & 0.01 & 0.51 \\
\hline Triglyceride & 0.073 & 0.091 & 0.081 & 0.079 & 0.012 & 0.90 & 0.53 & 0.44 \\
\hline Free glycerol & 0.222 & 0.206 & 0.356 & 0.304 & 0.035 & 0.01 & 0.36 & 0.63 \\
\hline NEFA & 0.082 & 0.091 & 0.144 & 0.141 & 0.026 & 0.05 & 0.92 & 0.82 \\
\hline Urea & 6.06 & 7.89 & 5.94 & 6.88 & 0.68 & 0.30 & 0.03 & 0.40 \\
\hline Insulin, $\mu \mathrm{IU} / \mathrm{mL}$ & 16.3 & 12.4 & 16.9 & 17.0 & 2.10 & 0.20 & 0.37 & 0.34 \\
\hline Leptin, ng/mL & 2.95 & 2.81 & 3.60 & 3.54 & 0.68 & 0.10 & 0.81 & 0.92 \\
\hline \multicolumn{9}{|c|}{ Jugular-mammary venous difference } \\
\hline Acetate & 0.631 & 0.428 & 0.247 & 0.272 & 0.118 & 0.05 & 0.48 & 0.40 \\
\hline$\beta$-hydroxybutyrate & 0.151 & 0.118 & 0.076 & 0.081 & 0.021 & 0.05 & 0.66 & 0.63 \\
\hline Lactate & 0.521 & 0.378 & 0.585 & 0.19 & 0.153 & 0.70 & 0.11 & 0.43 \\
\hline Glucose & 0.70 & 0.64 & 0.91 & 0.88 & 0.11 & 0.05 & 0.65 & 0.89 \\
\hline Total cholesterol & -0.09 & -0.14 & -0.05 & -0.50 & 0.16 & 0.33 & 0.14 & 0.21 \\
\hline Free cholesterol & -0.010 & -0.010 & 0.016 & -0.081 & 0.036 & 0.51 & 0.18 & 0.18 \\
\hline Phospholipid & -0.07 & -0.07 & -0.02 & -0.21 & 0.08 & 0.56 & 0.22 & 0.22 \\
\hline Triglyceride & 0.030 & 0.045 & 0.050 & 0.051 & 0.009 & 0.16 & 0.41 & 0.44 \\
\hline Free glycerol & 0.040 & 0.011 & -0.055 & 0.040 & 0.093 & 0.73 & 0.73 & 0.52 \\
\hline NEFA & -0.002 & -0.053 & 0.011 & -0.037 & 0.020 & 0.50 & 0.04 & 0.96 \\
\hline Urea & -0.050 & 0.003 & 0.001 & -0.0003 & 0.003 & 0.57 & 0.06 & 0.02 \\
\hline Insulin & -0.983 & -0.299 & 3.667 & 2.237 & 1.810 & 0.07 & 0.84 & 0.57 \\
\hline Leptin & 0.124 & -0.154 & 0.562 & -0.126 & 0.401 & 0.58 & 0.26 & 0.62 \\
\hline
\end{tabular}

${ }^{1} P$ value for the effect of concentrate level (Conc), oil, and the interaction of concentrate and oil. 
Table 3. Concentration of 12- to 22-carbon fatty acids [not including 18:1, 18:2, or 18:3 isomers] in jugular blood plasma from Holstein cows fed a high- (H) or low- (L) concentrate diet without supplemental oil (HC, LC), or supplemented at $3 \%$ of DM with linseed oil (HCO, LCO).

\begin{tabular}{|c|c|c|c|c|c|c|c|c|}
\hline \multirow[b]{2}{*}{ Fatty Acid } & \multicolumn{4}{|c|}{ Diets } & \multirow[b]{2}{*}{ SEM } & \multicolumn{3}{|c|}{ Effect $^{1}$} \\
\hline & $\mathrm{LC}$ & LCO & $\mathrm{HC}$ & $\mathrm{HCO}$ & & Conc & Oil & Conc $\times$ Oil \\
\hline & & $\mu \mathrm{g}$ & $\mathrm{nL}$ & & & & & \\
\hline $12: 0$ & 1.10 & 0.95 & 1.17 & 1.37 & 0.27 & 0.36 & 0.94 & 0.52 \\
\hline $14: 0$ & 14.3 & 10.7 & 12.6 & 15.7 & 1.3 & 0.22 & 0.86 & 0.02 \\
\hline cis $9-14: 1$ & 0.48 & 0.34 & 0.44 & 0.44 & 0.09 & 0.77 & 0.44 & 0.41 \\
\hline $15: 0$ & 15.4 & 16.8 & 13.9 & 18.0 & 1.2 & 0.86 & 0.02 & 0.19 \\
\hline iso-15:0 & 0.4 & 0.4 & 0.2 & 0.2 & 0.02 & 0.01 & 0.28 & 0.55 \\
\hline anteiso-15:0 & 19.0 & 24.4 & 12.6 & 15.4 & 1.3 & 0.01 & 0.01 & 0.26 \\
\hline $16: 0$ & 202 & 207 & 204 & 232 & 12 & 0.30 & 0.22 & 0.37 \\
\hline iso-16:0 & 7.79 & 7.45 & 5.73 & 5.87 & 0.55 & 0.01 & 0.75 & 0.39 \\
\hline trans $9-16: 1+i s o-17: 0$ & 7.90 & 5.02 & 5.78 & 5.28 & 0.74 & 0.23 & 0.04 & 0.14 \\
\hline cis $9-16: 1$ & 24.3 & 60.1 & 24.4 & 42.6 & 5.8 & 0.07 & 0.01 & 0.07 \\
\hline $17: 0$ & 17.5 & 16.2 & 18.6 & 19.0 & 1.2 & 0.13 & 0.71 & 0.48 \\
\hline cis $10-17: 1$ & 1.37 & 1.84 & 0.91 & 1.02 & 0.11 & 0.01 & 0.02 & 0.14 \\
\hline $18: 0$ & 308 & 451 & 301 & 364 & 20 & 0.04 & 0.01 & 0.05 \\
\hline 19:0 & 0.43 & 0.67 & 0.52 & 1.02 & 0.17 & 0.15 & 0.03 & 0.36 \\
\hline $20: 0$ & 6.95 & 1.27 & 4.44 & 1.03 & 3.57 & 0.71 & 0.23 & 0.75 \\
\hline cis $11-20: 1$ & 0.09 & 0.08 & 0.41 & 0.06 & 0.07 & 0.05 & 0.02 & 0.03 \\
\hline $20: 3 n-3$ & 43.6 & 45.5 & 45.1 & 44.9 & 3.0 & 0.84 & 0.71 & 0.65 \\
\hline $20: 3 n-6$ & 48.3 & 35.7 & 45.5 & 35.2 & 5.1 & 0.57 & 0.01 & 0.69 \\
\hline $20: 4 n-6$ & 16.9 & 16.2 & 20.7 & 24.7 & 1.4 & 0.01 & 0.24 & 0.10 \\
\hline $20: 5 n-3$ & 1.31 & 1.71 & 1.21 & 1.77 & 0.19 & 0.94 & 0.03 & 0.67 \\
\hline $22: 5 n-3$ & 0.09 & 0.03 & 0.13 & 0.08 & 0.01 & 0.01 & 0.01 & 0.64 \\
\hline Total $^{2}$ & 2108 & 2810 & 2122 & 2960 & 113 & 0.49 & 0.01 & 0.57 \\
\hline
\end{tabular}

${ }^{1} P$ value for the effect of concentrate level (Conc), oil, and the interaction of concentrate and oil.

${ }^{2}$ Total $=$ sum of 12:0 to 22:5n-3 (including 18:1, 18:2, and 18:3 isomers).

was added to the high-concentrate diet (Table 4). through 15 of the carbon chain were greater $(P<0.05)$ Among individual isomers, concentration of trans-18:1 in response to high-concentrate diets (Table 4). Linseed with double bonds in positions 5 through 11 and 13 oil supplementation resulted in greater $(P<0.05)$ con-

Table 4. Concentration of 18:1 isomers in jugular blood plasma from Holstein cows fed a high- $(\mathrm{H})$ or low(L) concentrate diet without supplemental oil (HC, LC), or supplemented at 3\% of DM with linseed oil (HCO, $\mathrm{LCO})$.

\begin{tabular}{|c|c|c|c|c|c|c|c|c|}
\hline \multirow[b]{2}{*}{ Isomer } & \multicolumn{4}{|c|}{ Diets } & \multirow[b]{2}{*}{ SEM } & \multicolumn{3}{|c|}{ Effect $^{1}$} \\
\hline & $\mathrm{LC}$ & LCO & $\mathrm{HC}$ & $\mathrm{HCO}$ & & Conc & Oil & Conc $\times$ Oil \\
\hline \multirow{2}{*}{\multicolumn{9}{|c|}{ 18:1 trans }} \\
\hline & & & & & & & & \\
\hline 4 & 1.33 & 1.49 & 1.39 & 2.02 & 0.21 & 0.11 & 0.04 & 0.19 \\
\hline 5 & 0.28 & 0.59 & 0.57 & 1.87 & 0.33 & 0.04 & 0.03 & 0.16 \\
\hline $6+7+8$ & 1.40 & 3.66 & 3.33 & 6.92 & 0.74 & 0.01 & 0.01 & 0.39 \\
\hline 9 & 1.22 & 3.27 & 2.94 & 5.74 & 0.64 & 0.01 & 0.01 & 0.56 \\
\hline 10 & 1.43 & 3.93 & 8.44 & 19.3 & 4.2 & 0.01 & 0.10 & 0.31 \\
\hline 11 & 13.7 & 35.9 & 19.2 & 91.7 & 8.0 & 0.05 & 0.01 & 0.12 \\
\hline 12 & 2.06 & 7.53 & 4.12 & 8.51 & 1.00 & 0.15 & 0.01 & 0.59 \\
\hline $13+14$ & 9.31 & 22.3 & 12.1 & 29.9 & 1.42 & 0.01 & 0.01 & 0.08 \\
\hline 15 & 1.57 & 7.10 & 3.26 & 9.29 & 0.72 & 0.02 & 0.01 & 0.71 \\
\hline 16 & 1.79 & 6.43 & 2.80 & 6.19 & 0.66 & 0.53 & 0.01 & 0.32 \\
\hline Total & 34.1 & 92.1 & 58.3 & 181 & 19.2 & 0.01 & 0.01 & 0.10 \\
\hline \multicolumn{9}{|l|}{$18: 1 \mathrm{cis}$} \\
\hline 9 & 153 & 212 & 146 & 176 & 20 & 0.28 & 0.04 & 0.46 \\
\hline 11 & 13.4 & 11.4 & 17.3 & 23.1 & 2.3 & 0.01 & 0.40 & 0.09 \\
\hline 12 & 4.31 & 13.7 & 9.78 & 27.8 & 5.30 & 0.06 & 0.01 & 0.38 \\
\hline 13 & 0.91 & 1.19 & 1.18 & 2.23 & 0.35 & 0.08 & 0.08 & 0.29 \\
\hline 15 & 3.02 & 4.95 & 3.57 & 10.8 & 1.04 & 0.01 & 0.01 & 0.01 \\
\hline Total & 174 & 242 & 178 & 239 & 21.1 & 0.82 & 0.01 & 0.78 \\
\hline
\end{tabular}

${ }^{1} P$ value for the effect of concentrate level (Conc), oil, and the interaction of concentrate and oil. 
Table 5. Concentration of 18:2 and 18:3 isomers in jugular blood plasma from Holstein cows fed a high$(\mathrm{H})$ or low- (L) concentrate diet without supplemental oil (HC, LC), or supplemented at 3\% of DM with linseed oil (HCO, LCO).

\begin{tabular}{|c|c|c|c|c|c|c|c|c|}
\hline \multirow[b]{2}{*}{ Isomer $^{2}$} & \multicolumn{4}{|c|}{ Diets } & \multirow[b]{2}{*}{ SEM } & \multicolumn{3}{|c|}{ Effect $^{1}$} \\
\hline & $\mathrm{LC}$ & LCO & $\mathrm{HC}$ & $\mathrm{HCO}$ & & Conc & Oil & Conc $\times$ Oil \\
\hline & & $-\mu_{\xi}$ & $\mathrm{mL}$ & & & & & \\
\hline \multicolumn{9}{|l|}{ 18:2 nonconjugated } \\
\hline $\operatorname{cis} 9, \operatorname{cis} 12$ & 925 & 1060 & 1029 & 1263 & 54 & 0.01 & 0.01 & 0.37 \\
\hline cis 9, trans 12 & 0.98 & 1.71 & 0.88 & 1.70 & 0.28 & 0.80 & 0.01 & 0.85 \\
\hline cis 9, trans 13 & 6.89 & 7.89 & 5.45 & 4.12 & 2.09 & 0.24 & 0.94 & 0.59 \\
\hline $\operatorname{trans} 9, \operatorname{cis} 12$ & 0.13 & 0.92 & 0.84 & 2.34 & 0.34 & 0.01 & 0.01 & 0.27 \\
\hline trans 9, trans 12 & 0.12 & 0.22 & 0.31 & 1.45 & 0.39 & 0.10 & 0.14 & 0.22 \\
\hline $\operatorname{trans} 11, c i s 15$ & 1.36 & 3.92 & 1.56 & 28.1 & 6.57 & 0.08 & 0.04 & 0.08 \\
\hline Total & 935 & 1078 & 1039 & 1305 & 54 & 0.01 & 0.01 & 0.28 \\
\hline \multicolumn{9}{|l|}{ 18:2 conjugated } \\
\hline cis 9, trans 11 & 1.84 & 4.83 & 2.06 & 9.48 & 1.49 & 0.10 & 0.01 & 0.14 \\
\hline trans 11, cis $13+$ cis 9, cis 11 & 0.10 & 0.09 & 0.19 & 0.05 & 0.03 & 0.40 & 0.03 & 0.05 \\
\hline $\operatorname{trans} 9$, cis 11 & 0.07 & 0.08 & 0.00 & 0.03 & 0.03 & 0.08 & 0.61 & 0.83 \\
\hline cis 10, cis 12 & 0.04 & 0.18 & 0.00 & 0.17 & 0.02 & 0.28 & 0.01 & 0.51 \\
\hline trans 11, trans 13 & 7.03 & 13.8 & 3.34 & 7.62 & 0.87 & 0.01 & 0.01 & 0.17 \\
\hline trans,trans & 0.17 & 0.17 & 0.19 & 0.30 & 0.10 & 0.44 & 0.54 & 0.55 \\
\hline Total & 9.24 & 19.2 & 5.78 & 17.6 & 2.01 & 0.22 & 0.01 & 0.62 \\
\hline \multicolumn{9}{|l|}{$18: 3$} \\
\hline cis $9, \operatorname{cis} 12, \operatorname{cis} 15$ & 184 & 432 & 99 & 359 & 25 & 0.01 & 0.01 & 0.80 \\
\hline cis 9, trans 12, cis 15 & 2.14 & 15.5 & 4.66 & 6.00 & 3.07 & 0.28 & 0.03 & 0.07 \\
\hline cis 9, trans, 12, trans 15 & 0.37 & 1.96 & 0.41 & 2.88 & 0.41 & 0.26 & 0.01 & 0.30 \\
\hline trans 9, trans 12, trans 15 & 7.45 & 18.8 & 5.67 & 10.78 & 5.37 & 0.35 & 0.13 & 0.55 \\
\hline Total & 206 & 462 & 115 & 379 & 25.7 & 0.01 & 0.01 & 0.89 \\
\hline
\end{tabular}

${ }^{1} P$ value for the effect of concentrate level (Conc), oil, and the interaction of concentrate and oil.

${ }^{2}$ Cis 9 ,cis 15-18:2, cis11,cis13-18:2, and trans10,cis12-18:2 were not detectable in plasma.

centrations of trans4-18:1 through trans9-18:1, and trans11-18:1 through trans16-18:1. Feeding linseed oil increased $(P<0.05)$ total cis-18:1 concentration by an average of $40 \%$, and this response was mostly due to increases in oleic acid.

\section{Plasma 18:2 and 18:3 Isomer Concentrations}

Linoleic acid (97 to $99 \%$ of total nonconjugated 18:2) concentration was greater $(P<0.05)$ in cows fed highconcentrate diets and it increased $(P<0.05)$ further with linseed oil (Table 5). Trans11,cis15-18:2 increased (concentrate $\times$ oil interaction, $P=0.08$ ) drastically with the high-concentrate diet plus linseed oil. Linseed oil resulted in greater total CLA $(+10.9 \mu \mathrm{g} / \mathrm{mL})$. Concentration of cis9,trans11-18:2 increased $(P<0.05)$ when linseed oil was fed. Feeding low-concentrate diets resulted in greater $(P<0.05)$ concentrations of trans 11 , trans 13 18:2 (the main CLA isomer in plasma), which were increased $(P<0.05)$ further with linseed oil. Total concentration of 18:3 isomers was greater $(P<0.05)$ in cows fed low-concentrate diets. Linseed oil supplementation increased $(P<0.05)$ 18:3 isomers 2 (LCO) to 3 (HCO) times. $\alpha$-Linolenic acid was the primary 18:3 isomer. Cis9,cis15-18:2, cis11,cis13-18:2, and trans10,cis1218:2 were not detectable in plasma.

\section{Milk Total Fatty Acid Yields}

Cows fed high-concentrate diets had lower yields of 4:0, 6:0, 8:0, 14:0, and 16:0 (Table 6). Yields of 8:0, 10:0, 12:0, 14:0, and 16:0 decreased $(P<0.05)$ in response to feeding linseed oil. Reductions in yield of 4:0 to 16:0 (mostly synthesized de novo) accounted for 76 (without oil) to $34 \%$ (with oil) of the decrease in total milk fatty acid yield in response to feeding high-concentrate diets. Stearic acid yield increased (concentrate $\times$ oil interaction, $P<0.05)$ more markedly with the low-concentrate diet plus linseed oil. Yields of cis9-10:1, cis9-14:1, and cis9-16:1 (products of $\Delta^{9}$-desaturation) decreased $(P<$ $0.05)$ with supplemental linseed oil.

\section{Yields of 18:1 Isomers}

Oleic acid accounted for 85 to $94 \%$ of total cis-18:1 isomers (Table 7). Responses to linseed oil varied with concentrate level. Whereas feeding LCO vs. LC increased total cis-18:1 by $110 \%$, feeding $\mathrm{HCO}$ vs. HC reduced yield by $27 \%$ (concentrate $\times$ oil interaction, $P$ $<0.05$ ). Total trans-18:1 yield increased (concentrate $\times$ oil interaction, $P<0.05$ ) more markedly with the lowconcentrate diet plus linseed oil (Table 7). Yields of trans $4-18: 1$, trans5-18:1, trans6+7+8-18:1 trans 12 18:1, trans $13+14-18: 1$, and trans $16-18: 1$ were greater 
Table 6. Yield of 4- to 22-carbon [not including 18:1, 18:2, or 18:3 isomers] fatty acids in milk fat from Holstein cows fed a high- (H) or low- (L) concentrate diet without supplemental oil (HC, LC), or supplemented at $3 \%$ of DM with linseed oil (HCO, LCO).

\begin{tabular}{|c|c|c|c|c|c|c|c|c|}
\hline \multirow[b]{2}{*}{ Fatty acid } & \multicolumn{4}{|c|}{ Diets } & \multirow[b]{2}{*}{ SEM } & \multicolumn{3}{|c|}{ Effect $^{1}$} \\
\hline & $\mathrm{LC}$ & LCO & $\mathrm{HC}$ & $\mathrm{HCO}$ & & Conc & Oil & Conc $\times$ Oil \\
\hline & & & & & & & & \\
\hline $4: 0$ & 22.2 & 28.0 & 20.4 & 14.3 & 2.37 & 0.01 & 0.94 & 0.01 \\
\hline $6: 0$ & 17.3 & 16.4 & 16.0 & 7.83 & 2.06 & 0.01 & 0.12 & 0.04 \\
\hline 8:0 & 11.3 & 10.2 & 10.8 & 4.68 & 1.49 & 0.06 & 0.03 & 0.11 \\
\hline $10: 0$ & 25.8 & 17.4 & 24.5 & 11.5 & 3.42 & 0.21 & 0.02 & 0.33 \\
\hline cis $9-10: 1$ & 2.47 & 1.72 & 2.14 & 0.78 & 0.28 & 0.02 & 0.01 & 0.22 \\
\hline $11: 0$ & 0.55 & 0.31 & 0.95 & 0.34 & 0.23 & 0.33 & 0.08 & 0.42 \\
\hline $12: 0$ & 29.2 & 18.1 & 27.2 & 13.5 & 4.02 & 0.29 & 0.02 & 0.53 \\
\hline $13: 0$ & 1.80 & 1.16 & 2.08 & 0.98 & 0.39 & 0.89 & 0.03 & 0.51 \\
\hline $14: 0$ & 85.7 & 70.1 & 72.4 & 42.3 & 9.85 & 0.05 & 0.05 & 0.42 \\
\hline $8: 0$ to $14: 0^{2}$ & 152 & 138 & 135 & 70.0 & 22 & 0.11 & 0.04 & 0.39 \\
\hline iso-14:0 & 0.94 & 1.03 & 0.53 & 0.35 & 0.08 & 0.01 & 0.55 & 0.11 \\
\hline cis $9-14: 1$ & 7.36 & 4.73 & 6.39 & 3.76 & 0.87 & 0.18 & 0.01 & 0.99 \\
\hline $15: 0$ & 9.13 & 7.47 & 9.09 & 6.57 & 1.31 & 0.28 & 0.14 & 0.30 \\
\hline iso-15:0 & 2.52 & 2.98 & 1.39 & 0.94 & 0.16 & 0.01 & 0.98 & 0.02 \\
\hline anteiso-15:0 & 5.30 & 5.76 & 3.79 & 2.57 & 0.34 & 0.01 & 0.28 & 0.03 \\
\hline $16: 0$ & 192 & 124 & 147 & 90.2 & 10.6 & 0.02 & 0.01 & 0.90 \\
\hline iso-16:0 & 2.11 & 2.30 & 1.57 & 0.95 & 0.20 & 0.01 & 0.27 & 0.05 \\
\hline trans $9-16: 1+i s o-17: 0$ & 3.5 & 4.0 & 3.5 & 3.7 & 0.5 & 0.14 & 0.05 & 0.17 \\
\hline cis $9-16: 1$ & 9.26 & 6.02 & 8.44 & 5.27 & 1.47 & 0.37 & 0.01 & 0.93 \\
\hline $17: 0$ & 5.09 & 4.01 & 4.78 & 2.80 & 0.57 & 0.10 & 0.05 & 0.25 \\
\hline cis $10-17: 1$ & 1.45 & 1.24 & 1.47 & 0.95 & 0.24 & 0.49 & 0.09 & 0.43 \\
\hline 18:0 & 45.3 & 110 & 33.9 & 38.7 & 9.37 & 0.01 & 0.01 & 0.01 \\
\hline 19:0 & 0.66 & 1.74 & 0.54 & 0.47 & 0.42 & 0.12 & 0.25 & 0.19 \\
\hline $20: 0$ & 0.82 & 0.35 & 0.60 & 0.22 & 0.10 & 0.10 & 0.01 & 0.66 \\
\hline cis $11-20: 1$ & 0.64 & 0.78 & 0.49 & 0.39 & 0.07 & 0.01 & 0.81 & 0.11 \\
\hline $21: 0$ & 0.31 & 0.54 & 0.21 & 0.15 & 0.10 & 0.03 & 0.39 & 0.16 \\
\hline $20: 3 n-3$ & 0.61 & 0.40 & 0.65 & 0.29 & 0.10 & 0.62 & 0.01 & 0.32 \\
\hline $20: 3 n-6$ & 0.39 & 0.49 & 0.25 & 0.18 & 0.04 & 0.01 & 0.64 & 0.06 \\
\hline $20: 4 n-6$ & 0.85 & 0.54 & 0.93 & 0.36 & 0.11 & 0.60 & 0.01 & 0.21 \\
\hline $20: 5 n-3$ & 0.44 & 0.42 & 0.31 & 0.23 & 0.05 & 0.01 & 0.15 & 0.35 \\
\hline cis 13, cis $16-22: 2$ & 0.043 & 0.031 & 0.035 & 0.018 & 0.004 & 0.04 & 0.01 & 0.35 \\
\hline $22: 4 n-6$ & 0.09 & 0.05 & 0.08 & 0.15 & 0.06 & 0.44 & 0.85 & 0.37 \\
\hline $22: 5 n-3$ & 0.57 & 0.21 & 0.72 & 0.36 & 0.09 & 0.11 & 0.01 & 0.98 \\
\hline $22: 6 n-3$ & 0.05 & 0.46 & 0.04 & 0.59 & 0.29 & 0.82 & 0.13 & 0.81 \\
\hline Total $^{3}$ & 707 & 843 & 622 & 481 & 60 & 0.01 & 0.71 & 0.07 \\
\hline
\end{tabular}

${ }^{1} P$ value for the effect of concentrate level (Conc), oil, and the interaction of concentrate and oil.

${ }^{2}$ Sum of even-numbered saturated fatty acids.

${ }^{3}$ Total $=$ sum of 4:0 to 22:6n-3 (including unidentified fatty acids, 18:1, 18:2, and 18:3 isomers).

(concentrate $\times$ oil interaction, $P<0.10$ ) with linseed oil addition primarily to the low-concentrate diet. Yield of trans10-18:1 was 3 to 5 times greater in response to high-concentrate diets. Linseed oil resulted in greater $(P<0.05)$ yields of trans9-18:1, trans11-18:1, and trans15-18:1.

\section{Yields of 18:2 and 18:3 Isomers}

Feeding linseed oil resulted in increases $(P<0.05)$ of 51 (HCO) and $80 \%$ (LCO) in the yield of nonconjugated isomers of 18:2, except for cis9,cis12-18:2 (Table 8). Trans11,cis15-18:2 alone accounted for 20 (LCO) and $35 \%$ ( $\mathrm{HCO}$ ) of the increase in total yield of nonconjugated 18:2 isomers in response to linseed oil. Yields of cis9,trans12-18:2 and cis9,trans13-18:2 were substan- tially increased (concentrate $\times$ oil interaction, $P<0.05$ ) with linseed oil addition to the low-concentrate diet primarily. Feeding linseed oil increased yields of total CLA by 120 to $169 \%$ (Table 8). Cis 9,trans 11-18:2 accounted for 80 to $91 \%$ of total conjugated 18:2 isomers regardless of diet. Its yield, however, increased by an average of $147 \%$ with linseed oil. Trans11,cis $13-$ + cis9,cis11-18:2 and trans11,trans 13-18:2 yields were increased to a greater extent (concentrate $\times$ oil interaction, $P<0.05)$ with the low-concentrate diet plus linseed oil.

Feeding linseed oil increased $(P<0.05)$ yields of total $18: 3$ isomers by $63 \%$. As expected, this response was largely due to the greater $(P<0.05)$ yield of $\alpha$-linolenic acid provided in the diet, which accounted for $88 \%$ of total 18:3 in milk fat. Yield of trans9,trans12,trans 15 - 
Table 7. Yield of 18:1 isomers in milk fat from Holstein cows fed a high- (H) or low- (L) concentrate diet without supplemental oil (HC, LC), or supplemented at $3 \%$ of DM with linseed oil (HCO, LCO).

\begin{tabular}{|c|c|c|c|c|c|c|c|c|}
\hline \multirow[b]{2}{*}{ Isomer } & \multicolumn{4}{|c|}{ Diets } & \multirow[b]{2}{*}{ SEM } & \multicolumn{3}{|c|}{ Effect $^{1}$} \\
\hline & $\mathrm{LC}$ & $\mathrm{LCO}$ & $\mathrm{HC}$ & $\mathrm{HCO}$ & & Conc & Oil & Conc $\times$ Oil \\
\hline & & & & & & & & \\
\hline \multicolumn{9}{|l|}{ 18:1 trans } \\
\hline 4 & 0.10 & 0.36 & 0.19 & 0.22 & 0.04 & 0.45 & 0.01 & 0.01 \\
\hline 5 & 0.07 & 0.25 & 0.17 & 0.22 & 0.03 & 0.34 & 0.01 & 0.06 \\
\hline $6+7+8$ & 1.34 & 4.48 & 2.51 & 3.50 & 0.40 & 0.81 & 0.01 & 0.02 \\
\hline 9 & 0.97 & 2.42 & 1.42 & 2.81 & 0.35 & 0.22 & 0.01 & 0.94 \\
\hline 10 & 2.00 & 4.41 & 10.3 & 13.0 & 3.15 & 0.01 & 0.37 & 0.95 \\
\hline 11 & 7.93 & 27.2 & 8.18 & 21.3 & 2.80 & 0.33 & 0.01 & 0.29 \\
\hline 12 & 1.44 & 5.33 & 2.10 & 2.99 & 0.40 & 0.05 & 0.01 & 0.01 \\
\hline $13+14$ & 2.43 & 20.4 & 3.80 & 12.30 & 3.05 & 0.10 & 0.01 & 0.05 \\
\hline 15 & 0.78 & 6.44 & 1.15 & 2.02 & 1.38 & 0.17 & 0.04 & 0.11 \\
\hline 16 & 1.16 & 5.81 & 1.39 & 2.37 & 0.38 & 0.01 & 0.01 & 0.01 \\
\hline Total & 18.2 & 77.1 & 31.2 & 55.7 & 7.16 & 0.53 & 0.01 & 0.02 \\
\hline \multicolumn{9}{|l|}{$18: 1 \mathrm{cis}$} \\
\hline 9 & 108 & 201 & 92.8 & 69.1 & 22.4 & 0.01 & 0.11 & 0.02 \\
\hline 11 & 4.79 & 5.51 & 6.44 & 5.89 & 0.71 & 0.18 & 0.91 & 0.38 \\
\hline 12 & 1.01 & 2.49 & 2.51 & 2.94 & 0.55 & 0.09 & 0.10 & 0.34 \\
\hline 13 & 0.49 & 2.31 & 0.65 & 0.57 & 0.74 & 0.30 & 0.26 & 0.22 \\
\hline 15 & 0.45 & 9.98 & 1.41 & 2.86 & 3.63 & 0.40 & 0.15 & 0.28 \\
\hline Total & 115 & 241 & 104 & 76.3 & 31.8 & 0.02 & 0.15 & 0.04 \\
\hline
\end{tabular}

${ }^{1} P$ value for the effect of concentrate level (Conc), oil, and the interaction of concentrate and oil.

Table 8. Yield of 18:2 and 18:3 isomers in milk fat from Holstein cows fed a high- $(\mathrm{H})$ or low- (L) concentrate diet without supplemental oil (HC, LC), or supplemented at 3\% of DM with linseed oil (HCO, LCO).

\begin{tabular}{|c|c|c|c|c|c|c|c|c|}
\hline \multirow[b]{2}{*}{ Isomer } & \multicolumn{4}{|c|}{ Diets } & \multirow[b]{2}{*}{ SEM } & \multicolumn{3}{|c|}{ Effect $^{1}$} \\
\hline & $\mathrm{LC}$ & $\mathrm{LCO}$ & $\mathrm{HC}$ & $\mathrm{HCO}$ & & Conc & Oil & Conc $\times$ Oil \\
\hline & & - & & & & & & \\
\hline \multicolumn{9}{|l|}{ 18:2 nonconjugated } \\
\hline cis $9, \operatorname{cis} 12$ & 11.4 & 11.7 & 15.4 & 11.1 & 1.7 & 0.28 & 0.21 & 0.17 \\
\hline cis 9, cis 15 & 1.02 & 1.20 & 0.91 & 0.79 & 0.12 & 0.05 & 0.81 & 0.28 \\
\hline cis 9, trans 12 & 0.41 & 1.69 & 0.78 & 1.23 & 0.19 & 0.78 & 0.01 & 0.02 \\
\hline cis 9, trans 13 & 0.87 & 4.29 & 1.44 & 3.08 & 0.43 & 0.38 & 0.01 & 0.03 \\
\hline $\operatorname{trans} 9, \operatorname{cis} 12$ & 0.19 & 0.56 & 0.33 & 0.99 & 0.15 & 0.07 & 0.01 & 0.31 \\
\hline $\operatorname{trans} 9, \operatorname{trans} 12$ & 0.02 & 0.27 & 0.19 & 0.85 & 0.18 & 0.05 & 0.02 & 0.25 \\
\hline $\operatorname{trans} 11$, cis 15 & 0.76 & 6.71 & 1.41 & 12.9 & 2.34 & 0.16 & 0.01 & 0.25 \\
\hline Total & 14.7 & 26.4 & 20.5 & 31.0 & 3.61 & 0.14 & 0.01 & 0.86 \\
\hline \multicolumn{9}{|l|}{ 18:2 conjugated } \\
\hline cis 9, trans 11 & 4.39 & 11.3 & 4.98 & 11.8 & 1.76 & 0.73 & 0.01 & 0.98 \\
\hline trans 11, cis $13+$ cis 9, cis 11 & 0.07 & 0.58 & 0.12 & 0.26 & 0.05 & 0.01 & 0.01 & 0.01 \\
\hline $\operatorname{trans} 9$, cis 11 & 0.15 & 0.78 & 0.25 & 0.18 & 0.29 & 0.41 & 0.36 & 0.26 \\
\hline cis 10, cis 12 & 0.19 & 0.27 & 0.16 & 0.12 & 0.05 & 0.06 & 0.69 & 0.21 \\
\hline cis 11, cis 13 & 0.10 & 0.06 & 0.00 & 0.00 & 0.04 & 0.03 & 0.48 & 0.48 \\
\hline trans 10, cis 12 & 0.05 & 0.14 & 0.07 & 0.05 & 0.04 & 0.42 & 0.39 & 0.18 \\
\hline trans 11, trans 13 & 0.16 & 0.75 & 0.13 & 0.33 & 0.06 & 0.01 & 0.01 & 0.01 \\
\hline trans,trans & 0.17 & 0.30 & 0.21 & 0.26 & 0.04 & 0.97 & 0.01 & 0.15 \\
\hline Total & 5.28 & 14.2 & 5.92 & 13.0 & 1.77 & 0.86 & 0.01 & 0.57 \\
\hline \multicolumn{9}{|l|}{$18: 3$} \\
\hline cis 9, cis 12, cis 15 & 5.53 & 8.42 & 4.72 & 7.63 & 0.84 & 0.35 & 0.01 & 0.99 \\
\hline cis 9, trans 12, cis 15 & 0.13 & 0.16 & 0.01 & 0.04 & 0.02 & 0.01 & 0.25 & 0.96 \\
\hline cis 9, trans, 12, trans 15 & 0.11 & 0.18 & 0.04 & 0.10 & 0.02 & 0.01 & 0.01 & 0.61 \\
\hline trans 9, trans 12, trans 15 & 0.23 & 1.28 & 0.19 & 0.53 & 0.08 & 0.01 & 0.01 & 0.01 \\
\hline Total & 6.25 & 10.2 & 5.17 & 8.35 & 0.86 & 0.10 & 0.01 & 0.63 \\
\hline
\end{tabular}

${ }^{1} P$ value for the effect of concentrate level (Conc), oil, and the interaction of concentrate and oil. 
Table 9. Percentages of selected fatty acids in milk fat from Holstein cows fed a high- (H) or low- (L) concentrate diet without supplemental oil (HC, LC), or supplemented at 3\% of DM with linseed oil (HCO, LCO).

\begin{tabular}{|c|c|c|c|c|c|c|c|c|}
\hline \multirow[b]{2}{*}{ Fatty acids } & \multicolumn{4}{|c|}{ Diets } & \multirow[b]{2}{*}{ SEM } & \multicolumn{3}{|c|}{ Effect $^{1}$} \\
\hline & $\mathrm{LC}$ & LCO & $\mathrm{HC}$ & $\mathrm{HCO}$ & & Conc & Oil & Conc $\times$ Oil \\
\hline & \multicolumn{4}{|c|}{ - $\mathrm{g} / 100 \mathrm{~g}$ of total fatty acids -} & & & & \\
\hline $8: 0-14: 0^{2}$ & 23.3 & 15.4 & 22.8 & 15.1 & 1.0 & 0.73 & 0.01 & 0.92 \\
\hline $16: 0$ & 29.4 & 17.2 & 25.7 & 18.7 & 0.9 & 0.08 & 0.01 & 0.04 \\
\hline 18:0 & 7.05 & 14.8 & 6.17 & 8.07 & 1.0 & 0.01 & 0.01 & 0.01 \\
\hline total trans-18:1 & 2.69 & 9.02 & 5.00 & 12.1 & 1.21 & 0.02 & 0.01 & 0.69 \\
\hline trans $10-18: 1$ & 0.28 & 0.52 & 1.66 & 2.84 & 0.46 & 0.01 & 0.07 & 0.10 \\
\hline trans11-18:1 & 1.12 & 3.23 & 1.32 & 4.53 & 0.53 & 0.27 & 0.01 & 0.09 \\
\hline trans $13+14-18: 1$ & 0.34 & 2.42 & 0.61 & 2.56 & 0.50 & 0.42 & 0.01 & 0.21 \\
\hline total cis-18:1 & 17.7 & 31.8 & 18.3 & 16.7 & 4.5 & 0.14 & 0.20 & 0.11 \\
\hline cis9-18:1 & 15.3 & 23.8 & 14.9 & 14.4 & 2.8 & 0.55 & 0.26 & 0.04 \\
\hline total nonconjugated $18: 2$ & 2.25 & 3.37 & 3.54 & 7.06 & 0.84 & 0.01 & 0.01 & 0.15 \\
\hline $\operatorname{trans} 11, \operatorname{cis} 15-18: 2$ & 0.11 & 0.80 & 0.23 & 2.84 & 0.50 & 0.08 & 0.01 & 0.05 \\
\hline total conjugated $18: 2$ & 0.82 & 1.82 & 1.04 & 3.00 & 0.36 & 0.15 & 0.01 & 0.31 \\
\hline cis 9, trans $11-18: 2$ & 0.62 & 1.34 & 0.81 & 2.54 & 0.36 & 0.13 & 0.02 & 0.12 \\
\hline total $18: 3$ & 0.96 & 1.30 & 0.91 & 1.86 & 0.18 & 0.14 & 0.01 & 0.09 \\
\hline
\end{tabular}

${ }^{1} P$ value for the effect of concentrate level (Conc), oil, and the interaction of concentrate and oil.

${ }^{2}$ Sum of even-numbered saturated fatty acids.

18:3 was more than 6 times greater due to feeding LCO compared with $\mathrm{HC}$ (concentrate $\times$ oil interaction, $P=$ $0.01)$.

\section{Percentage of Selected Fatty Acids in Milk Fat}

Significant concentrate $\times$ oil interactions were found for the percentage of a number of fatty acids (Table 9 ). A more pronounced decrease $(P<0.05)$ in percentage of 16:0 was observed with LCO vs. LC. Percentage of 18:0 and cis9-18:1 increased markedly with LCO $(P<$ $0.05)$; whereas percentages of trans $10-18: 1$, trans 11 18:1, trans 11 ,cis 15-18:2, and total 18:3 isomers was increased $(P<0.10)$ to a greater extent with HCO. Cis9,trans11-18:2 percentage also tended $(P=0.12)$ to increase more markedly when HCO was fed.

\section{Correlation Analysis}

Iso-14:0, iso-15:0, and 18:0 percentages had the highest positive correlations with milk fat percentage (Table 10). Trans 9-18:1 and trans10-18:1 had the most negative correlations with milk fat percentage among trans18:1, but cis11-18:1, trans 9 , cis 11-CLA, and 18:2n-6 had the highest overall negative correlations. Percentages of cis $15-18: 1$, trans $6+7+8-18: 1$, trans $9-18: 1$, trans $11-$ 18:1, and trans $12-18: 1$ (also trans $4-18: 1$, trans5-18:1, cis13-18:1, data not shown, and total trans-18:1, $\mathrm{r}=$ -0.97) in milk fat were highly negatively correlated with the percentage of even-numbered $4: 0$ to $16: 0$ in milk fat. Among all 18:2 isomers, cis9,trans11-18:2, cis9,trans $13-18: 2$, cis9,trans $12-18: 2$, and trans 11 ,cis $13-+$ cis9,cis 11-18:2 had the most negative correlations with percentage of 4:0 to $16: 0$ in milk fat.

\section{DISCUSSION}

\section{Plasma Metabolites and Hormones}

In the present study, reduced plasma acetate concentration in cows fed high-concentrate diets was associated with lower molar concentration of acetate in ruminal fluid (Ueda et al., 2003). Although ruminal butyrate concentration was not affected by concentrate level (Ueda et al., 2003), recent data suggest that acetate and butyrate production ( $\mathrm{mol} / \mathrm{d}$ ) may be decreased by high dietary concentrate (Sutton et al., 2003). Such responses could account for the observed lower jugular plasma concentration and jugular-mammary venous difference for acetate and BHBA (Table 3). These changes may partly contribute to the effect of dietary concentrate level on milk fat yield. Others have shown that, despite an estimated 34\% more acetate and 35\% more butyrate produced in the rumen of cows fed highconcentrate diets, there was a significant depression in milk fat yield (Oba and Allen, 2003). In the present study, the absence of an interaction effect on plasma acetate and BHBA and the low correlation between jugular-mammary venous differences for those metabolites and either milk fat percentage $(r=0.12$ to 0.24$)$ or percentage of 4:0-16:0 $(\mathrm{r}=0.28$ to 0.33$)$ clearly suggests that other factors are needed to fully explain milk fat depression due to high-concentrate diets.

Responses in plasma glucose with high-concentrate diets, but not NEFA or free glycerol, were consistent 
Table 10. Correlations $(\mathrm{n}=16 ; P \leq 0.05)$ among the percentage of 4:0-16:0 ${ }^{1}$ or milk fat and selected milk fatty acids.

\begin{tabular}{|c|c|c|c|}
\hline Fatty acid & Fat & Fatty acid & $4: 0-16: 0^{2}$ \\
\hline $\begin{array}{l}4: 0 \\
\text { iso-14:0 } \\
\text { iso-15:0 } \\
18: 0 \\
\text { trans } 9-18: 1 \\
\text { trans } 10-18: 1 \\
\text { cis } 11-18: 1 \\
\text { cis } 12-18: 1 \\
18: 2 \mathrm{n}-6 \\
\text { trans } 9, \text { trans } 12-18: 2 \\
\text { trans } 9, \text { cis } 12-18: 2 \\
\text { cis } 9, \text { trans } 11-18: 2 \\
\text { trans } 9, \text { cis } 11-18: 2 \\
\text { trans,trans-CLA } \\
18: 3 \mathrm{n}-3\end{array}$ & $\begin{array}{r}0.55 \\
0.82 \\
0.78 \\
0.66 \\
-0.65 \\
-0.70 \\
-0.85 \\
-0.62 \\
-0.82 \\
-0.63 \\
-0.65 \\
-0.56 \\
-0.73 \\
-0.58 \\
-0.53\end{array}$ & $\begin{array}{l}17: 0 \\
20: 0 \\
\text { trans } 6+7+8-18: 1 \\
\text { trans } 9-18: 1 \\
\text { trans } 11-18: 1 \\
\text { trans } 12-18: 1 \\
\text { cis } 15-18: 1 \\
\text { cis } 9, \text { trans } 13-18: 2 \\
\text { cis } 9, \text { trans } 12-18: 2 \\
\text { trans } 11, \text { cis } 15-18: 2 \\
\text { cis } 9, \text { trans } 11-18: 2 \\
\text { trans } 11, \text { cis } 13-18: 2 \\
\text { trans } 11, \text { trans } 13-18: 2 \\
\text { trans,trans-CLA } \\
18: 3 \mathrm{n}-3 \\
20: 4 \mathrm{n}-6\end{array}$ & $\begin{array}{r}0.71 \\
0.69 \\
-0.89 \\
-0.74 \\
-0.84 \\
-0.77 \\
-0.81 \\
-0.90 \\
-0.89 \\
-0.73 \\
-0.80 \\
-0.89 \\
-0.69 \\
-0.68 \\
-0.70 \\
0.78\end{array}$ \\
\hline
\end{tabular}

${ }^{1}$ Correlations were very similar if using concentrations of 8:0-14:0, which more closely reflects de novo fatty acid synthesis.

${ }^{2}$ Sum of even-numbered saturated fatty acids.

with previous results (Gaynor et al., 1995; Griinari et al., 1998). As in the present study, Gagliostro et al. (1991) reported greater concentrations of phospholipids and cholesterol in blood plasma from cows infused with rapeseed oil into the duodenum. Dietary unsaturated oils also increase concentrations of all plasma lipid fractions in blood (Loor et al., 2002b). It was previously shown that dry nonpregnant cows fed high levels of concentrate compared with underfed cows (Delavaud et al., 2002) had greater concentrations of plasma leptin, in agreement with the present study.

\section{Yields of Milk Fat, Stearic, and Oleic Acids}

High dietary concentrate:forage reduced milk fat percentage and yield (Table 1). Gaynor et al. (1995) and Kalscheur et al. (1997) found similar responses in cows fed $75 \%$ concentrate (DM basis) without added lipid. Others reported more pronounced reductions in milk fat percentage and yield when feeding high-concentrate diets plus corn or soybean oil (Griinari et al., 1998; Piperova et al., 2000; Peterson et al., 2003). We found a significant oil $\times$ concentrate interaction effect for milk fat yield. The positive response $(+20 \%)$ in cows fed LCO vs. LC was due to higher yields of 18:0 $(+65 \mathrm{~g} / \mathrm{d})$ and cis9-18:1 (+93 g/d) in milk (oil effect, Figure 1), which more than compensated for the decrease in yield of 8:0 to $14: 0(-14 \mathrm{~g} / \mathrm{d})$ plus 16:0 (-68 g/d) (oil effect, Figure 1). The negative response $(-21 \%)$ to linseed oil on milk fat yield in cows fed $\mathrm{HCO}$ vs. HC was due to lower yields of $8: 0$ to $14: 0(-65 \mathrm{~g} / \mathrm{d}), 16: 0(-57 \mathrm{~g} / \mathrm{d})$, and cis918:1 (-24 g/d) (oil effect, Figure 1).

The marked increase in milk oleic acid yield with LCO compared with HCO may have been associated with greater duodenal flow of 18:0 (+258 g/d) (Loor et al., 2004) and plasma availability for desaturation in the mammary gland. The higher concentration of 18:0 in plasma (Table 3) and yield in milk fat with LCO vs. LC (Table 6; oil effect, Figure 1) support this suggestion. In contrast, yield and percentage of 18:0 increased very little when feeding HCO (oil effect, Figure 1; Table 9), which was partly associated with a smaller increase $(+112 \mathrm{~g} / \mathrm{d})$ in duodenal flow of 18:0 compared with LCO (Loor et al., 2004). Reduced milk fat output with HCO may have been associated with a lack of endogenously synthesized oleic acid for triglyceride formation (Loor and Herbein, 2003). Alternatively, substitution of trans-18:1 for oleic acid could increase the melting point of milk fat and inhibit its secretion from mammary cells (Chilliard et al., 2000). The importance of 18:0 in the process of milk fat secretion is supported by a relatively high correlation between 18:0 and milk fat content (Table 10) and the coordinated responses in 18:0 and oleic acid yields (Figure 1; see also Loor and Herbein, 2003 and Chilliard et al., 2003b).

\section{Trans10-18:1 and Milk Fat Yield}

Addition of high-linoleic acid oil (1 to $4 \%$ of DM) to high-concentrate diets resulted in pronounced milk fat depression along with increased trans10-18:1 in milk fat (Griinari et al., 1998; Peterson et al., 2003). Across a substantial number of published studies (Figure 2), data clearly suggests that increased percentage of trans 10-18:1 in milk fat is positively correlated with milk fat depression in cows fed high-concentrate diets with or without unsaturated oils, or mixed diets with various levels of fish oil. Greater trans10-18:1 percent- 


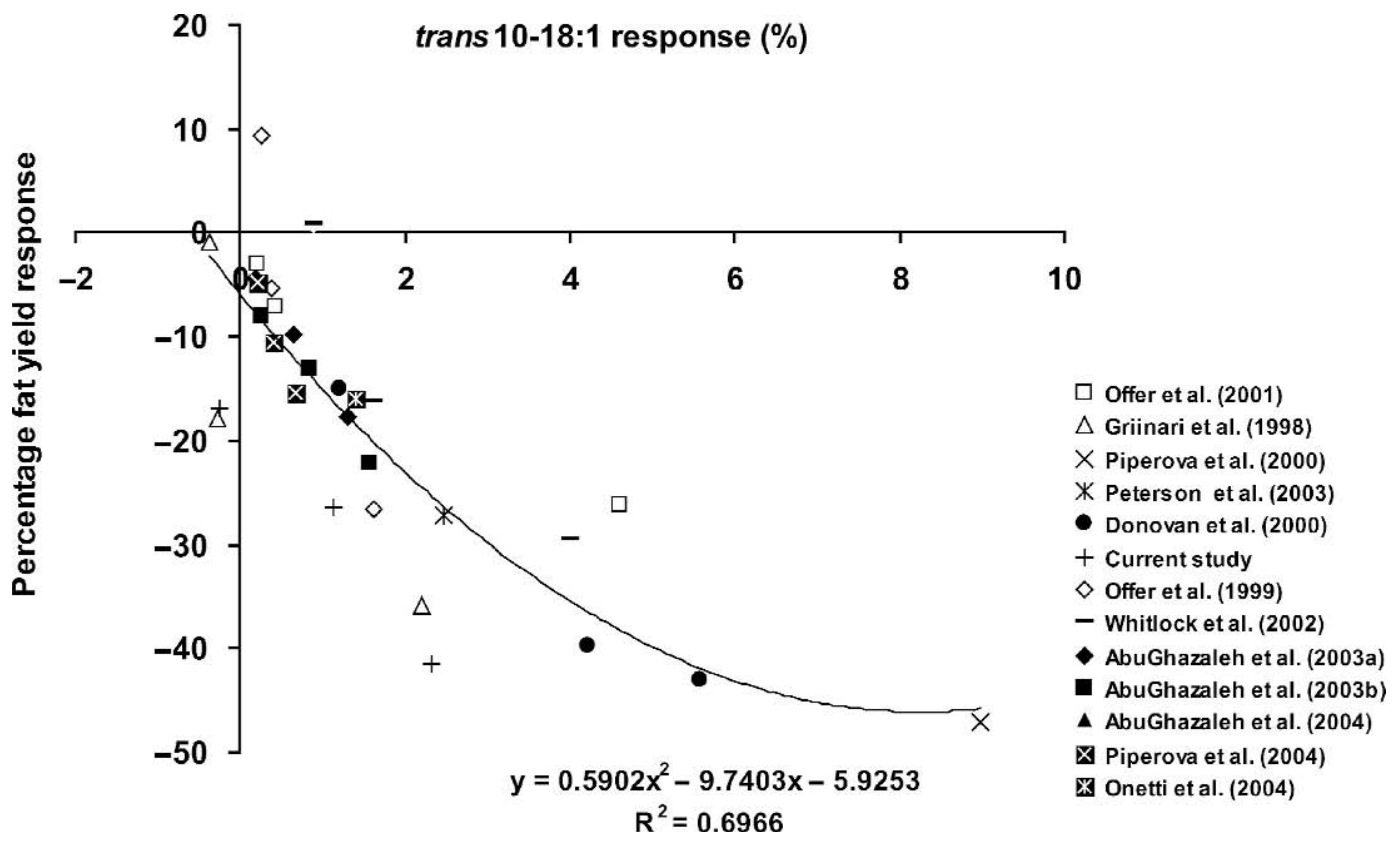

Figure 2. Relationship ( $\mathrm{n}=31$ comparisons) among the change in percentage of trans10-18:1 in milk fat with the corresponding change in fat yield observed in the current and other published studies. Responses were calculated by comparison with unsupplemented control diets (Offer et al., 1999, 2001; Piperova et al., 2000; Piperova et al., 2004 only for control vs. Ca-salts of trans-18:1 treatments; Peterson et al., 2003; Donovan et al., 2000; Whitlock et al., 2002; AbuGhazaleh et al., 2003a,b; AbuGhazaleh et al., 2004; Onetti et al., 2004 only for corn silage vs. corn silage + tallow) or by comparing the treatment with highest milk fat yield against the remaining 3 in factorial designs (current study and Griinari et al., 1998). Fitting a linear equation $(y=-5.5312 \times-8.7709)$ through the data resulted in a value for $R^{2}=0.63$.

age and reduced mRNA abundance for a number of lipogenic enzymes were observed due to feeding a highconcentrate diet plus linoleic acid rich oils (1 to $5 \%$ of DM) (Piperova et al., 2000; Peterson et al., 2003). Our results show that feeding high-concentrate diets without linseed oil essentially did not change uptake of 18carbon fatty acids (e.g., 18:0, total trans-18:1, total cis18:1, total $18: 2$, and total $18: 3$; Tables 6,7 , and 8; concentrate effect for 18:0 in Figure 1), but it decreased synthesis of 14:0 (Table 6) and probably 16:0 (concentrate effect, Figure 1). The responses in 14:0 and 16:0 (Table 6, oil effect in Figure 1), but also 4:0, 6:0, and 8:0 (Table 6), to high-concentrate diets were more marked with linseed oil. Although abomasal infusions of trans10,cis12-18:2 caused milk fat depression (Baumgard et al., 2000; Loor and Herbein, 2003), few feeding studies have shown increases in trans10,cis12-18:2 in cows with depressed milk fat and they were very modest (less than $0.10 \%$ of total milk fatty acids; Piperova et al., 2000; Peterson et al., 2003). We speculate that trans 10 ,cis $12-18: 2$ is likely not the only fatty acid capable of inhibiting milk fat synthesis.

Trans10-18:1 in response to high-concentrate plus unsaturated oil accounted for 36\% of total trans-18:1 in the study of Griinari et al. (1998), for 59\% in Piperova et al. (2000), for $67 \%$ in Offer et al. (2001), for $43 \%$ in
Peterson et al. (2003), or for $24 \%$ in the present study. Piperova et al. (2000) used corn silage as the sole forage compared with alfalfa hay by Griinari et al. (1998) or grass hay in the present study. Different proportions of trans 10-18:1 in milk fat may be associated with higher dietary starch content (32 to 45\%) in those studies compared with ours (23\%), and the fact that they fed linoleic acid-rich oils or fish oil. We previously speculated (Loor et al., 2004) that changes in ruminal microbial populations, buffering capacity, or increased passage rate with higher dietary starch intake may play a role in enhancing trans-18:1 production in the rumen. In goats, feeding linseed oil or high-oleic sunflower oil increased trans 10-18:1 when the basal diet was corn silage (20\% starch content in DMI) but not with alfalfa hay (16\% starch content in DMI) (Chilliard et al., 2003a). Although Figure 2 clearly suggests that trans $10-18: 1$ is a good candidate to explain milk fat depression in cows, it should be noted that, in the present study, several other isomers of 18:1 and 18:2 were more closely related to either milk fat percentage and 4:0-16:0 percentage (Table 10 and next section).

\section{Rumen-Derived Trans Fatty Acids and De Novo Fatty Acid Synthesis}

Compared with the level of dietary concentrate, which decreased yields of 4:0, 6:0, 14:0, 16:0, odd, and 
branched-chain fatty acids with 14 to 16 carbons mainly (Table 6), linseed oil alone caused greater reductions in the yield (Table 6) and percentage (Table 9) of most fatty acids (8:0 to 16:0) synthesized de novo in mammary. Sunflower oil supplementation to grazing cows resulted in a comparable response (Kay et al., 2004). Chilliard et al. (2000) discussed how dietary manipulation could reduce the percentage of saturated fatty acids synthesized de novo. More recently, it was shown that pure trans11-18:1 compared with oleic acid reduced activity of acetyl-CoA carboxylase and fatty acid synthase (EC 2.3.1.85) in bovine mammary cell cultures (Jayan and Herbein, 2000). Although speculative given the small number of observations, data from correlations (Table 10) raise the possibility that cis11-18:1, cis1518:1, and several trans-18:1 isomers may be good candidates (in addition to trans10-18:1) as potential inhibitors of milk fat synthesis. Among octadecadienoic acids, those that may have been in part synthesized via desaturation of their corresponding 18:1 isomers (cis11-, trans11-, trans12-, and trans13-), could be involved in the reduction of de novo fatty acid synthesis and contribute to an overall decrease in fat synthesis. Good candidates also could be among those fatty acids that increased the most in blood plasma with HCO (for example, trans6-18:1 through trans11-18:1, trans13+1418:1, and trans11,cis 15-18:2; Tables 4 and 5). Further studies with purified preparations are needed to unravel the role of the different isomers in the regulation of milk fat secretion in ruminants.

\section{Linolenic Acid Intake and Biohydrogenation Intermediates in Plasma and Milk}

In terms of actual amounts, responses in trans1118:1, trans $13+14-18: 1, \quad$ cis $11-18: 1, \quad$ cis $15-18: 1$, trans 11,cis 15-18:2, and cis9,trans11-18:2 due to feeding HCO were more pronounced in plasma (Tables 4 and 5) than in milk (Tables 7 and 8). The major isomers secreted in milk fat were trans11-18:1 (up to $27 \mathrm{~g} / \mathrm{d}$ ), trans13+14-18:1 (up to $20 \mathrm{~g} / \mathrm{d}$ ), cis9,trans 13-18:2 (up to $4 \mathrm{~g} / \mathrm{d}$ ), and trans11,cis15-18:2 (up to $13 \mathrm{~g} / \mathrm{d}$ ), whereas the yield of $\alpha$-linolenic acid remained below $9 \mathrm{~g} / \mathrm{d}$. These data are in agreement with duodenal flows of 18:3n3 and its biohydrogenation intermediates (Loor et al., 2004), and with milk fatty acid responses to increased 18:3n-3 intake (Loor et al., 2002a, 2003; Chilliard et al., 2003c; Kay et al., 2004).

The increase in milk total CLA yield (Table 8) and percentage (Table 9) in response to linseed oil is in agreement with previous milk data (Chilliard et al., 2000 ) and with their greater duodenal flow plus that of trans11-18:1 (Loor et al., 2004). In blood plasma, trans11,trans13-18:2 was the major CLA followed by cis9,trans11-18:2. Trans11,trans13-18:2 in milk is likely derived from the circulation and its low concentration in milk fat, coupled with the low correlation between plasma concentration and percentage in milk fat (data not shown), may indicate that it was not readily taken up by the mammary gland (Loor and Herbein, 2003).

No changes were found in the percentage or yield of trans 10,cis12-CLA, and it was not correlated to changes in trans10-18:1 seen due to concentrate-linseed oil interactions. Thus, contrary to previous studies (Piperova et al., 2000; Peterson et al., 2003) but, in agreement with correlation analyses by Precht et al. (2002) and Chilliard et al. (2003b), this CLA isomer was not associated with changes in milk fat content $(\mathrm{r}=0.23)$ or yield in our study.

\section{Apparent Desaturation of Fatty Acids via $\Delta^{9}$-Desaturase}

Correlation among percentage of trans11-18:1 $(\mathrm{r}=$ $0.94)$ and percentage of cis 9, trans $11-18: 2(\mathrm{r}=0.97)$ in plasma with their respective percentages in milk fat was high as previously shown (Loor et al., 2002b). Basal activity of $\Delta^{9}$-desaturase was not affected (e.g., no effect on desaturation ratios of cis $9-14: 1 / 14: 0$ and cis $9-16: 1 /$ 16:0). A role for $\Delta^{9}$-desaturase in desaturation of trans18:1 isomers (Bauman and Griinari, 2003) is supported by the close associations we observed among increases of substrates and products in plasma and milk fat. Yield of cis9,trans11-18:2, cis9,trans12-18:2, cis9,trans1318:2 in milk increased by up to $2.6,4$, and 4.9 times, respectively, in response to linseed oil, which corresponded with greater duodenal flow (Loor et al., 2004) and availability of trans $11-18: 1$, trans $12-18: 1$, and trans 13+14-18:1 in blood plasma (Table 4). From current and previous results (Chilliard et al., 2003ac), it appears that cis 9, trans 13-18:2 may be formed via desaturation of trans 13+14-18:1 which, based on its duodenal flow (Loor et al., 2004), is derived primarily during biohydrogenation of dietary 18:3n-3.

\section{CONCLUSIONS}

Milk fat yield decreased by feeding the high-concentrate diet but was much lower with the combination of linseed oil and high dietary concentrate. Yields of 4:0, 6:0, branched-chain fatty acids, 18:0, and cis9-18:1 in milk fat were drastically reduced but yields of various trans- and cis-18:1 isomers (not including trans 10-18:1), trans 11,cis 15-18:2, and trans isomers of 18:3 increased when linseed oil was fed in combination with high dietary concentrate. Results, however, indicate that influx of trans10-18:1 may affect key regulatory steps in 
the pathway for de novo lipogenesis in mammary tissue when cows are fed high-concentrate diets without unsaturated oils. Other trans-fatty acids and a decrease in oleic acid secretion, however, must be considered to fully explain the marked depression in milk fat yield with high-concentrate diets supplemented with unsaturated oils. Lower 18:0 availability for endogenous synthesis of cis9-18:1 appears to be a primary factor reducing milk fat synthesis when feeding high-concentrate diets and unsaturated oils. It remains to be determined whether a single isomer or a combination of isomers is directly responsible for reduced mammary lipogenesis. The changes in milk fatty acid profile due to oil supplementation, especially when fed with high-concentrate diets, should modify the nutritional value of dairy products.

\section{ACKNOWLEDGMENTS}

Authors acknowledge the assistance of J. P. Pezant and the team of Les Cèdres Experimental Unit for feeding, milking, blood sampling, and caring for cows, as well as P. Capitan, M. Tourret, and C. Delavaud for help in laboratory analyses. This study was partly funded by the French Ministry of Research (project "Increase by nutritional means in CLA and trans fatty acid content in milk and beef" of the "Aliment Qualité Sécurité" program).

\section{REFERENCES}

AbuGhazaleh, A. A., D. J. Schingoethe, A. R. Hippen, and K. F. Kalscheur. 2003a. Milk conjugated linoleic acid response to fish oil supplementation of diets differing in fatty acid profiles. J. Dairy Sci. 86:944-953.

AbuGhazaleh, A. A., D. J. Schingoethe, A. R. Hippen, and K. F. Kalscheur. 2003b. Conjugated Linoleic acid and vaccenic acid in rumen, plasma, and milk of cows fed fish oil and fats differing in saturation of 18 carbon fatty acids. J. Dairy Sci. 86:3648-3660.

AbuGhazaleh, A. A., D. J. Schingoethe, A. R. Hippen, and K. F. Kalscheur. 2004. Conjugated linoleic acid increases in milk when cows fed fish meal and extruded soybeans for an extended period of time. J. Dairy Sci. 87:1758-1766.

Association of Official Analytical Chemists. 1997. Official Methods of Analysis. 16th ed. AOAC Int., Gaithersburg, MD.

Bauman, D. E., and J. M. Griinari. 2003. Nutritional regulation of milk fat synthesis. Annu. Rev. Nutr. 23:203-227.

Baumgard, L. H., B. A. Corl, D. A. Dwyer, A. Saebo, and D. E. Bauman. 2000. Identification of the conjugated linoleic acid isomer that inhibits milk fat synthesis. Am. J. Physiol. 278:R179-R184.

Chilliard, Y., A. Ferlay, and M. Doreau. 2001. Effect of different types of forages, animal fat or marine oils in cow's diet on milk fat secretion and composition, especially conjugated linoleic acid (CLA) and polyunsaturated fatty acids. Livest. Prod. Sci. 70:31-48.

Chilliard, Y., A. Ferlay, R. Mansbridge, and M. Doreau. 2000. Ruminant milk fat plasticity: Nutritional control of saturated, polyunsaturated, trans and conjugated fatty acids. Ann. Zootech. 49:181-205.

Chilliard, Y., A. Ferlay, J. Rouel, and G. Lamberet. 2003a. A review of nutritional and physiological factors affecting goat milk lipid synthesis and lipolysis. J. Dairy Sci. 86:1751-1770.
Chilliard, Y., J. Rouel, P. Capitan, J. M. Chabosseau, K. RaynalLjutovac, and A. Ferlay. 2003b. Correlations between milk fat content and fatty acid composition in goats receiving different combinations of forages and lipid supplements. Page 343 in Book of Abstracts, 54th Annu. Mtg. Eur. Assoc. Anim. Prod., Rome, Italy. Y. van der Honing, ed. Wageningen Acad. Publ., Wageningen, The Netherlands.

Chilliard, Y., J. Rouel, J. M. Chabosseau, P. Capitan, P. Gaborit, and A. Ferlay. 2003c. Interactions between raygrass preservation and linseed oil supplementation on goat milk yield and composition, including trans and conjugated fatty acids. Page 343 in Book of Abstracts, 54th Annu. Mtg. Eur. Assoc. Anim. Prod., Rome, Italy. Y. van der Honing, ed. Wageningen Acad. Publ., Wageningen, The Netherlands.

Christie, W. W., J. L. Sebedio, and P. Juaneda. 2001. A practical guide to the analysis of conjugated linoleic acid. Inform 12:147-156.

Delavaud, C., A. Ferlay, Y. Faulconnier, F. Bocquier, G. Kann, and Y. Chilliard. 2002. Plasma leptin concentration in adult cattle: Effects of breed, adiposity, feeding level, and meal intake. J. Anim. Sci. 80:1317-1328.

Donovan, D. C., D. J. Schingoethe, R. J. Baer, J. Ryali, A. R. Hippen, and S. T. Franklin. 2000. Influence of dietary fish oil on conjugated linoleic acid and other fatty acids in milk fat from lactating dairy cows. J. Dairy Sci. 83:2620-2628.

Doreau, M., Y. Chilliard, H. Rulquin, and D. I. Demeyer. 1999. Manipulation of milk fat in dairy cows. Pages 81-109 in Recent Advances in Animal Nutrition. P. C. Garnsworthy and J. Wiseman, ed. Nottingham Press, Nottingham, UK.

Ferlay, A., and Y. Chilliard. 1999. Effects of the infusion of nonselective beta-, and selective beta1- or beta2-adrenergic agonists, on body fat mobilisation in underfed or overfed non-pregnant heifers. Reprod. Nutr. Dev. 39:409-421.

Gagliostro, G., Y. Chilliard, and M. J. Davicco. 1991. Duodenal rapeseed oil infusion in early and midlactation cows. 3. Plasma hormones and apparent mammary uptake of metabolites. J. Dairy Sci. 74:1893-1903.

Gaynor, P. J., D. R. Waldo, A. V. Capuco, R. A. Erdman, L. W. Douglass, and B. B. Teter. 1995. Milk fat depression, the glycogenic theory, and trans-18:1 fatty acids. J. Dairy Sci. 78:2008-2015.

Griinari, J. M., D. A. Dwyer, M. A. McGuire, D. E. Bauman, D. L. Palmquist, and K. V. V. Nurmela. 1998. Trans-octadecenoic acids and milk fat depression in lactating cows. J. Dairy Sci. 81:1251-1261.

Jayan, G. C., and J. H. Herbein. 2000. Healthier dietary fat using trans-vaccenic acid. Nutr. Food Sci. 30:304-309.

Kalscheur, K. F., B. B. Teter, L. S. Piperova, and R. A. Erdman. 1997. Effect of dietary forage concentration and buffer addition on duodenal flow of trans-C18:1 fatty acids and milk fat production in dairy cows. J. Dairy Sci. 80:2104-2114.

Kay, J. K., T. R. Mackle, M. J. Auldist, N. A. Thomson, and D. E. Bauman. 2004. Endogenous synthesis of cis-9, trans-11 conjugated linoleic acid in dairy cows fed fresh pasture. J. Dairy Sci. 87:369-378.

Loor, J. J., and J. H. Herbein. 2003. Reduced fatty acid synthesis and desaturation due to exogenous trans 10,cis12-CLA in cows fed oleic or linoleic oil. J. Dairy Sci. 86:1354-1369.

Loor, J. J., J. H. Herbein, and C. E. Polan. 2002a. Trans 18:1 and 18:2 isomers in blood plasma and milk fat of grazing cows fed a grain supplement containing solvent-extracted or mechanically extracted soybean meal. J. Dairy Sci. 85:1197-1207.

Loor, J. J., L. E. Quinlan, A. B. P. A. Bandara, and J. H. Herbein. 2002b. Distribution of trans-vaccenic acid and cis9, trans11- conjugated linoleic acid (rumenic acid) in blood plasma lipid fractions and secretion into milk fat of Jersey cows fed canola or soybean oil. Anim. Res. 51:119-134.

Loor, J. J., F. D. Soriano, X. Lin, J. H. Herbein, and C. E. Polan. 2003. Grazing allowance after the morning or afternoon milking for lactating cows fed a total mixed ration (TMR) enhances vaccenic acid (trans11-18:1) and rumenic acid (cis9,trans11-18:2) in milk fat to different extents. Anim. Feed Sci. Technol. 109:105-119. 
Loor, J. J., K. Ueda, A. Ferlay, Y. Chilliard, and M. Doreau. 2004. Biohydrogenation, duodenal flows, and intestinal digestion of trans fatty acids and conjugated linoleic acids in response to dietary forage:concentrate ratio and linseed oil in dairy cows. J. Dairy Sci. 87:2472-2485.

Oba, M., and M. S. Allen. 2003. Effects of corn grain conservation method on feeding behavior and productivity of lactating dairy cows at two dietary starch concentrations. J. Dairy Sci. 86:174-183.

Offer, N. W., M. Marsden, J. Dixon, B. K. Speake, and F. E. Thacker. 1999. Effect of dietary fat supplements on levels of n-3 polyunsaturated fatty acids, trans acids and conjugated linoleic acid in bovine milk. Anim. Sci. 69:613-625.

Offer, N. W., M. Marsden, and R. H. Phipps. 2001. Effect of oil supplementation of a diet containing a high concentration of starch on levels of trans fatty acids and conjugated linoleic acids in bovine milk. Anim. Sci. 73:533-540.

Onetti, S. G., S. M. Reynal, and R. R. Grummer. 2004. Effect of alfalfa forage preservation method and particle length on performance of dairy cows fed corn silage-based diets and tallow. J. Dairy Sci. 87:652-664.

Peterson, D. G., E. A. Matitashvili, and D. E. Bauman. 2003. Dietinduced milk fat depression in dairy cows results in increased trans-10, cis-12 CLA in milk fat and coordinate suppression of mRNA abundance for mammary enzymes involved in milk fat synthesis. J. Nutr. 133:3098-3102.

Piperova, L. S., U. Moallem, B. B. Teter, J. Sampugna, M. P. Yurawecz, K. M. Morehouse, D. Luchini, and R. A. Erdman. 2004. Changes in milk fat in response to dietary supplementation with calcium salts of trans-18:1 or conjugated linoleic fatty acids in lactating dairy cows. J. Dairy Sci. 87:3836-3844.

Piperova, L. S., J. Sampugna, B. B. Teter, K. Kalscheur, M. Yurawecz, Y. Ku, K. Morehouse, and R. A. Erdman. 2002. Duodenal and milk trans octadecenoic acid and conjugated linoleic acid (CLA) isomers indicate postabsorptive synthesis is the predominant source of cis9-containing CLA in lactating dairy cows. J. Nutr. 132:1235-1241.

Piperova, L. S., B. B. Teter, I. Bruckental, J. Sampugna, S. E. Mills, M. P. Yurawecz, J. Fritsche, K. Ku, and R. A. Erdman. 2000. Mammary lipogenic enzyme activity, trans fatty acids and conjugated linoleic acids are related in lactating dairy cows fed a milk fat-depressing diet. J. Nutr. 130:2568-2574.

Precht, D., H. Hagemeister, W. Kanitz, and J. Voigt. 2002. Milk fat depression and the role of trans and CLA fatty acid isomers by feeding a high fiber diet with calcium soaps of fatty acids in early lactating dairy cows. Milchwissenschaft 57:518-522.

Sutton, J. D., M. S. Dhanoa, S. V. Morant, J. France, D. J. Napper, and E. Schuller. 2003. Rates of production of acetate, propionate, and butyrate in the rumen of lactating dairy cows given normal and low-roughage diets. J. Dairy Sci. 86:3620-3633.

Ueda, K., A. Ferlay, J. Chabrot, J. J. Loor, Y. Chilliard, and M. Doreau. 2003. Effect of linseed oil supplementation on ruminal digestion in dairy cows fed diets with different forage:concentrate ratios. J. Dairy Sci. 86:3999-4007.

Whitlock, L. A., D. J. Schingoethe, A. R. Hippen, K. F. Kalscheur, R. J. Baer, N. Ramaswamy, and K. M. Kasperson. 2002. Fish oil and extruded soybeans fed in combination increase conjugated linoleic acids in milk of dairy cows more than when fed separately. J. Dairy Sci. 85:234-243. 